\title{
VIOLENCIA DE GÉNERO ASOCIADO AL INICIO DE LAS RELACIONES SEXUALES EN LOS ADOLESCENTES DE LA I. E. MARÍA PARADO DE BELLIDO, SANTOYO EL AGUSTINO, JUNIO 2016
}

\author{
GENDER VIOLENCE ASSOCIATED WITH THE START OF SEXUAL RELATIONS IN ADOLESCENTS OF THE \\ I. E. MARÍA PARADO DE BELLIDO. SANTOYO. EL AGUSTINO, JUNE 2016. \\ Sandra Miranda-Vargas', Lucy Correa-López'1,2, Consuelo Luna-Muñoz ${ }^{1,2}$
}

\begin{abstract}
RESUMEN
Objetivos: Determinar la asociación de violencia de género y el inicio de las relaciones sexuales de estudiantes adolescentes I.E María Parado de Bellido, Santoyo, El Agustino, Lima, Junio 2016. Diseño: Descriptivo, transversal, relacional y analítico. Lugar: El Agustino. Santoyo, Lima. Métodos: Muestra no probabilística, población: 198 adolescentes de 11 a 18 años de edad, de ambos sexos. Análisis: Se aplica chi cuadrado, con nivel de significancia estadística $p=0.000<0.05$, Odds Ratio IC al 95\%. Resultados: Adolescentes que iniciaron su relación sexual tienen $(I C=95 \%, O R=6,8)$ veces la probabilidad de presentar riesgo de violencia de género, $46,4 \%$ de adolescentes que iniciaron su relación sexual presentaron violencia de género según tipo: primer lugar violencia familiar ( $\mathrm{IC}=95 \%, \mathrm{OR}=5,48)$, violencia sexual $(\mathrm{IC}=95 \%, \mathrm{OR}=3.73)$, violencia psicológica (IC=95\%, OR=2.41), violencia física ( $I C=95 \%, O R=1.88)$. Las variables con asociación significativa con violencia de género $(p=<0,05)$ : inicio de las RS, año que cursa, violencia psicológica, sexual y familiar. Las variables sexo, edad, lugar donde viven, con quién viven y violencia física no están asociadas a la violencia de género ( $\mathrm{p}=>$ $0,05)$. No son factor de riesgo $(O D<1)$ las variables: sexo, lugar donde viven, año que cursan. Variables $(O D>1)$ : Inicio RS, edad y con quien viven, son factores de riesgo de violencia de género. Conclusiones: Adolescentes que inician sus relaciones sexuales tienen mayor riesgo y está asociado a la violencia de género. El tipo de violencia de género como mayor factor de riesgo y estadísticas significativas de asociación: violencia familiar, seguida violencias sexual y psicológica.
\end{abstract}

Palabras clave: Adolescentes; Inicio de relación sexual; Violencia de género. (fuente: DeCS BIREME)

\begin{abstract}
Objective: To determine the association of gender violence and the beginning of the sexual relations of adolescent students I.E María Parado de Bellido, Santoyo, El Agustino, Lima, June 2016. Design: Descriptive, transverse, relational and analytical. Location: El Agustino. Santoyo, Lima. Methods: Non-probabilistic sample, population: 198 adolescents between 11 and 18 years of age, of both sexes. Analysis: Chi square is applied, with statistical significance level $p=0.000<0.05,95 \%$ Odds Ratio IC. Results: Adolescents who began their sexual relationship had a probability of presenting a risk of gender violence $(\mathrm{Cl}=95 \%, \mathrm{OR}=6.8), 46.4 \%$ of adolescents who started their sexual relations had gender violence according to type: first place $(\mathrm{Cl}=95 \%$, OR $=5.48)$, sexual violence $(\mathrm{Cl}=95 \%, \mathrm{OR}=3.73)$, psychological violence $(\mathrm{Cl}=95 \%, \mathrm{OR}=2.41)$, physical violence $=$ 1.88). Variables with a significant association with gender violence $(p=<0.05)$ : RS, current year, psychological, sexual and family violence. The variables gender, age, place where they live, who they live with and physical violence are not associated with gender violence $(p=>0.05)$. There are no risk factors $(O D<1)$ for the variables: sex, place where they live, year of study. Variables (OD> 1): RS initiation, age and with whom they live, are risk factors for gender violence. Conclusions: Adolescents who initiate their sexual relations are at greater risk and associated with gender violence. The type of gender violence as a major risk factor and significant association statistics: family violence, followed sexual and psychological violence.
\end{abstract}

Key words: Adolescents; Beginning of sexual relationship; gender violence. (source: MeSH NLM)

Facultad de Medicina Humana. Universidad Ricardo Palma. Lima . Perú.

${ }^{2}$ Instituto de Investigación en Ciencias Biomédicas. Facultad de Medicina Humana. Universidad Ricardo Palma. Lima. Perú.

Correspondencia: Sandra Miranda-Vargas Dirección: Jr. Santander 279, Pueblo Libre. Celular: 94587225. Correo : smirva@hotmail.com

Citar como: Sandra Miranda-Vargas, Lucy Correa-López, Consuelo Luna-Muñoz. Violencia de género asociado al inicio de las relaciones sexuales en los adolescentes de la I.E. María Parado de Bellido, Santoyo el Agustino, junio 2016 [Artículo Original]. Rev. Fac. Med. Hum. $2016 ; 16(3): 53-59$. DOI 10.25176/RFMH.v16.n3.653 


\section{INTRODUCCIÓN}

La violencia de género es un grave problema social visible debido a la mayor concientización de las víctimas y sus familias. La OMS en el estudio sobre Salud de la mujer y Violencia doméstica, realizado en 2005 precisó que en 10 países más de 700 millones de mujeres a nivel mundial han sido víctimas de violencia de género'.

A nivel mundial las y los adolescentes representan el $20 \%$ de la población total y de este $20 \%$, el $85 \%$ se encuentra en los países en vías de desarrollo. En América del Sur, el Perú es el cuarto país con mayor cantidad de habitantes después de Brasil, Colombia y Argentina ${ }^{2}$.

El inicio sexual está ligado a la violencia de género. Muchos adolescentes de todo el mundo tienen relaciones sexuales forzadas, matrimonio infantil, falta de acceso a métodos anticonceptivos y a la información sexual, embarazos no deseados sobre todo en los países en desarrollo en donde es evidente la desigualdad y el no respeto a los derechos del niño y adolescente pasan a las siguientes generaciones cuando las adolescentes en condición de pobreza son madres como consecuencia de esta situación ${ }^{3}$.

La violencia sexual, incluido el acoso sexual, ocurre con frecuencia en instituciones supuestamente "seguras", como las escuelas, donde los agresores incluyen compañeros o profesores. En estudios provenientes de diversas partes del mundo, con inclusión de África, Asia meridional y América Latina, se ha documentado que proporciones sustanciales de niñas dicen haber sufrido acoso y abuso sexuales en camino hacia la escuela o de regreso de esta, o bien en instalaciones de la escuela o la universidad, incluidos baños, aulas y dormitorios, y que los perpetradores eran compañeros o profesores ${ }^{3}$.

ENDES-Perú 2015 el 49\% de chicos y chicas reciben golpizas por parte de los padres, consideran esta práctica por los propios chicos como un método natural de disciplina y educación. Se estima que 8 de cada 10 casos de abuso sexual tienen como victimario a un miembro del entorno familiar y que, 6 de cada 10 embarazos en niñas de 11-14 años, son producto de incesto o violación ${ }^{4}$.

Los costos sociales y económicos de este problema son enormes y repercuten en toda la sociedad. Las adolescentes y mujeres pueden llegar a encontrarse aisladas e incapacitadas para estudiar y/o trabajar 5 . El propósito del presente estudio es evidenciar si existe asociación entre el inicio de las relaciones sexuales y la violencia de género en los adolescentes y determinar cuáles son factores de riesgo para adoptar medidas de prevención para esta población.

\section{MÉTODOS}

La investigación es de tipo observacional, transversal, relacional, analítico, prospectivo. La muestra en estudio estuvo constituida por 198 estudiantes adolescentes de ambos sexos de 11 a 18 años, estudiantes de $1^{\circ}$ a $5^{\circ}$ año de secundaria de la I.E María Parado de Bellido (Institución pública). Muestra: no probabilística los estudiantes que cumplían los criterios de inclusión .Técnica: Encuesta, instrumento: cuestionario. Medición de las variables: Se aplicó el análisis estadístico de confiabilidad el alfa de Cronbrach. Para determinar la validez de criterios se aplico (Prueba de K- R-20) prueba de Kuder Richarson -20, para el análisis bivariado se aplicó la prueba de Chi Cuadrado para evaluar la significancia estadística. Se aplicó la razón de momios (RM), razón de probabilidades (odds ratio (OR). En los cálculos estadísticos se utilizó el programa SPSS ${ }^{22}$.

\section{RESULTADOS}

Fueron evaluados 198 estudiantes de los cuales $47 \%$ eran mujeres y $53 \%$ varones (Gráfico № 1).

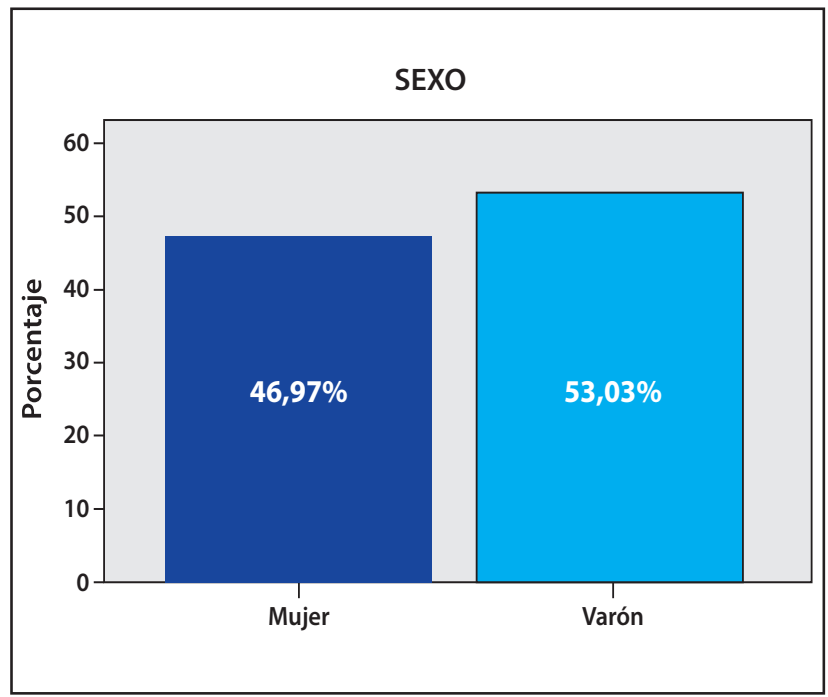

Gráfico 1. Sexo de los adolescentes de la I.E.M.P de Bellido.

Los estudiantes que presentaron violencia se describe en la tabla № 1, observándose que los alumnos de los dos primeros años, presentaron menor violencia de género en relación a los de los tres últimos años con mayor \% de violencia de género. 
Tabla 1. Año que cursan y violencia de género en los estudiantes.

Violencia de Género

\begin{tabular}{cccccc} 
& Año que cursa & & Presente & Ausente & Total \\
& 1ero. y 2 2 $^{\text {Secundaria }}$ & Recuento & 19 & 37 & 56 \\
& 3ero. a 5to & Recuento & 73 & $34,9 \%$ & $28,3 \%$ \\
& Secundaria & $\%$ & $79,3 \%$ & 69 & 142 \\
Total & & Recuento & 92 & $65,1 \%$ & $71,7 \%$ \\
& & $\%$ & $100,0 \%$ & 106 & 198 \\
\hline
\end{tabular}

El inicio de la relación sexual y la violencia de género en los estudiantes se describen en el gráfico №, 2 el grupo que ya inicio relaciones sexuales tiene 6.8 veces mayor probabilidad de riesgo de presentar violencia de género, en relación a aquellos que no iniciaron su relación sexual.

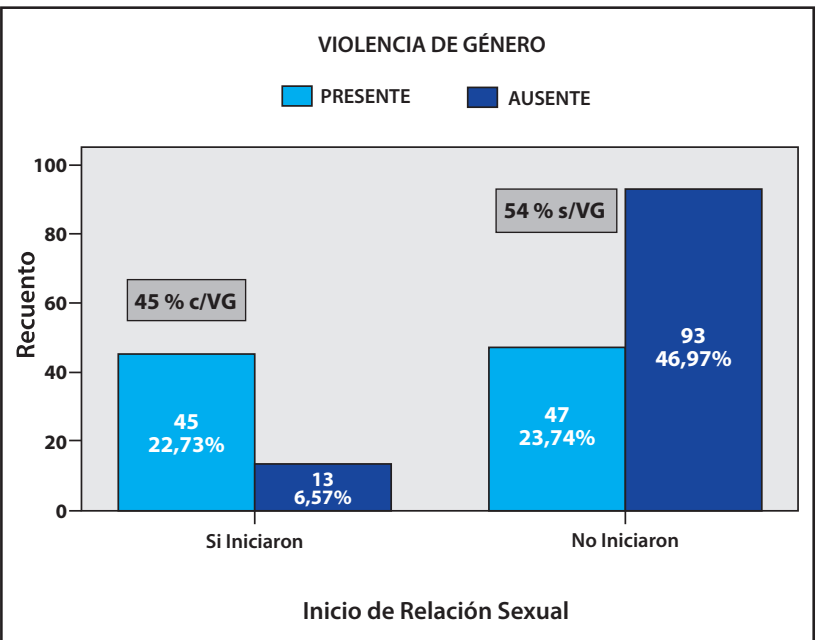

Gráfico 2. Inicio de Relaciones Sexuales y Violencia de Género.

En relación a la asociación de la violencia de género al inicio de las relaciones sexuales se observa en la campana de Gauss del gráfico № 3 de acuerdo al resultado obtenido con el proceso Chi Cuadrado para el grado de libertad de 6, corresponde a 3.84, que es menor al calculado 31.939; por lo tanto, la violencia de género está asociada al inicio de las relaciones sexuales en los estudiantes adolescentes de la Institución Educativa María Parado de Bellido. Santoyo. El Agustino, junio 2016.

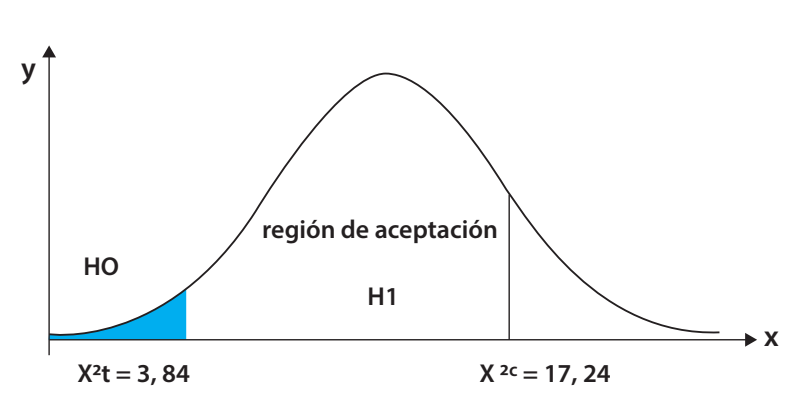

Gráfico 3. Violencia de género asociado al inicio de las relaciones sexuales en adolescentes de al I.E. María Parado de Bellido. Santoyo El Agustino 2016. X2t $=3.84 / \mathrm{X} 2 \mathrm{c}=31.939$
La relación de la edad con el inicio de las relaciones sexuales se observa en la tabla № 2 , conforme aumenta la edad los estudiantes tienen mayor probabilidad de riesgo de presentar violencia de género.

Tabla 2. Edad y violencia de género en las adolescentes de la I.E. María Parado de Bellido. Santoyo El Agustino Junio 2016.

\begin{tabular}{|c|c|c|c|c|}
\hline \multicolumn{5}{|c|}{ Violencia de Género } \\
\hline Edad & & Presente & Ausente & Total \\
\hline $\begin{array}{c}11 \text { a } 12 \\
\text { años }\end{array}$ & $\begin{array}{l}\text { Recuento } \\
\% \text { dentro de } \\
\text { VIOLENCIA DE } \\
\text { GÉNERO }\end{array}$ & $4,8 \%$ & $2,0 \%$ & $3,3 \%$ \\
\hline $\begin{array}{c}13 \text { a } 18 \\
\text { años }\end{array}$ & $\begin{array}{l}\text { Recuento } \\
\% \text { dentro de } \\
\text { VIOLENCIA DE } \\
\text { GÉNERO }\end{array}$ & $\begin{array}{c}40 \\
95,2 \%\end{array}$ & $\begin{array}{c}49 \\
98,0 \%\end{array}$ & $\begin{array}{c}89 \\
96,7 \%\end{array}$ \\
\hline Total & $\begin{array}{l}\text { Recuento } \\
\% \text { dentro de } \\
\text { VIOLENCIA DE } \\
\text { GÉNERO }\end{array}$ & $100,0 \%$ & $100,0 \%$ & $\begin{array}{c}92 \\
100,0 \%\end{array}$ \\
\hline
\end{tabular}

La descripción estadística de la forma de inicio de las relaciones sexuales se observa en la tabla № 3 los adolescentes que iniciaron voluntariamente su relación sexual tienen mayor probabilidad de riesgo y asociación significativa con la violencia de género que los que no iniciaron voluntariamente.

Tabla 3. Forma de inicio de las relaciones sexuales y violencia de género en los adolescentes de al I.E. María Parado de Bellido, Santoyo. El Agustino.

\begin{tabular}{ccccc}
\hline \multicolumn{5}{c}{ Violencia de Género } \\
$\begin{array}{c}\text { Forma de } \\
\text { Inicio de } \\
\text { Relación Sexual }\end{array}$ & & Presente & Ausente & Total \\
\hline Si & $\begin{array}{c}\text { Recuento } \\
\%\end{array}$ & 54 & 0 & 54 \\
\hline No & $\begin{array}{c}\text { Recuento } \\
\%\end{array}$ & $38,7 \%$ &, $0 \%$ & $27,3 \%$ \\
\hline Total & $\begin{array}{c}\text { Recuento } \\
\%\end{array}$ & $91,3 \%$ & $100,0 \%$ & $72,7 \%$ \\
\hline
\end{tabular}


En cuanto a la prevalencia del tipo de violencia de género en los estudiantes es $46.4 \%$, como se observa en el gráfico 7. La violencia psicológica es predominante seguida de la violencia sexual, en menor porcentaje los otros tipos de violencia, este hallazgo corrobora los informes de investigaciones internacionales.

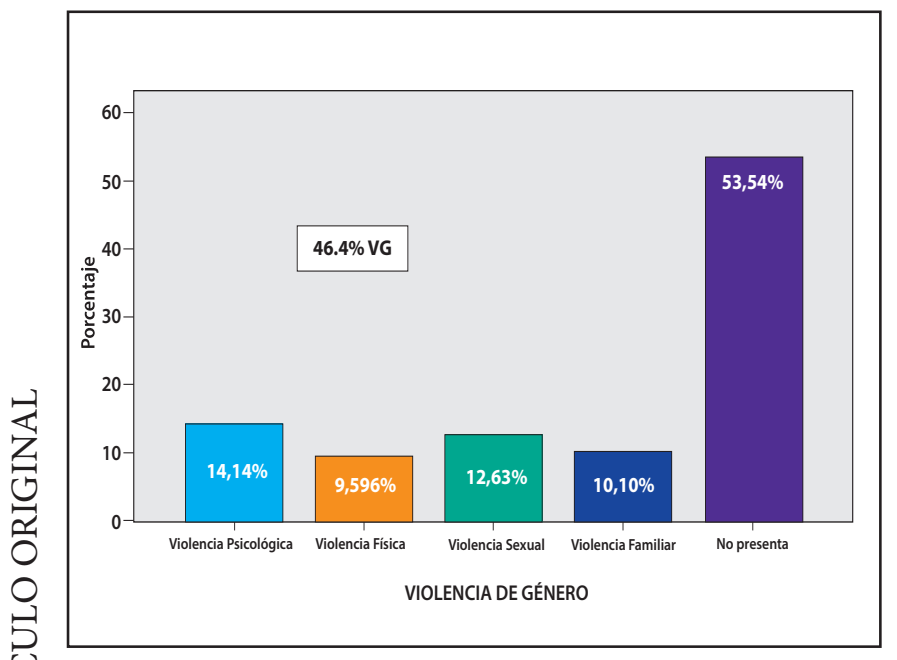

Gráfico 4. Tipos de violencia de género.

En la a estimación de riesgo entre el inicio de las relaciones sexuales y los tipos de violencia de genero en los adolescentes, en los tipos : familiar y sexual tienen 5,4 y 3,7 veces respectivamente mayor probabilidad de riesgo de violencia y tienen una asociación estadísticamente significativa; seguidos de los tipos de violencia psicológica y física .

Tabla 4. Estimación de riesgo entre el inicio de la relación sexual y tipos de violencia de género en adolescentes.

\begin{tabular}{ccccc}
\hline $\begin{array}{c}\text { Inicio de } \\
\text { Relaciones } \\
\text { Sexuales }\end{array}$ & \multicolumn{5}{c}{ Tipos de Violencia de Género } \\
\cline { 2 - 4 } & Psicologia & Física & Sexual & Familiar \\
\hline OR & 2,40 & 1,87 & 3,73 & 5,48 \\
\hline $\begin{array}{c}\text { IC } 95 \\
\%\end{array}$ & 1,063 &, 713 & 1,578 & 2,062 \\
\hline MIN & 5,450 & 4,037 & 8,824 & 14,611 \\
\hline $\begin{array}{c}\text { Chi } 2 \text { Valor } \mathrm{p} \\
<0,05\end{array}$ &, 032 &, 197 &, 002 &, 000 \\
\hline
\end{tabular}

Las variables con asociación significativa con la violencia de género se describen en la tabla No 5 son: inicio de las RS, año que cursa, violencia psicológica, sexual y familiar

Mientras que las variables sexo, edad, lugar donde viven, con quién viven y violencia física no están asociadas a la violencia de género.

No son factor de riesgo por tener OD $<1$ las variables sexo, lugar donde viven y año que cursan. Las variables con un OD >1: Inicio RS, edad, con quien viven, los 4 tipos de violencia tienen veces más la probabilidad de presentar el riesgo de violencia de género.

Tabla 5. Estimación de riesgo y asociación entre las diferentes variables y la violencia de género.

\begin{tabular}{|c|c|c|c|c|}
\hline \multicolumn{5}{|c|}{ Violencia de Género } \\
\hline \multirow[t]{2}{*}{ Variables } & \multirow[t]{2}{*}{ OR } & \multicolumn{2}{|c|}{ IC $95 \%$} & \multirow{2}{*}{$\begin{array}{c}\text { Chi2 Valor } \\
\text { p }\end{array}$} \\
\hline & & Minimo & Máximo & \\
\hline $\begin{array}{l}\text { Inicio de } \\
\text { relación } \\
\text { sexual }\end{array}$ & 6,84 & 3,368 & 13,930 & 0,000 \\
\hline Sexo & 0,970 & 0,426 & 2,210 & 0,942 \\
\hline Edad & 2,45 & ,214 & 28,011 & 0,458 \\
\hline $\begin{array}{l}\text { Lugar } \\
\text { donde vive }\end{array}$ & 0,194 & 0,21 & 1,800 & 0,113 \\
\hline $\begin{array}{l}\text { Año que } \\
\text { cursa }\end{array}$ & 235 & ,088 & ,628 & 0,003 \\
\hline $\begin{array}{l}\text { Con quien } \\
\text { vive }\end{array}$ & 1,12 &, 435 & 2,909 & 0,942 \\
\hline $\begin{array}{l}\text { Violencia } \\
\text { psicologica }\end{array}$ & 2,65 & 2,189 & 3,223 & 0,000 \\
\hline $\begin{array}{l}\text { Violencia } \\
\text { fisica }\end{array}$ & 2,45 & 2,055 & 2,925 & 0,187 \\
\hline $\begin{array}{l}\text { violencia } \\
\text { sexual }\end{array}$ & 2,52 & 2,141 & 3,114 & 0,000 \\
\hline $\begin{array}{l}\text { Violencia } \\
\text { familiar }\end{array}$ & 2,47 & 2,069 & 2,955 & 0,000 \\
\hline
\end{tabular}

\section{DISCUSIÓN}

La investigación halló que los estudiantes que inician su relación sexual tienen un riesgo de 6,89 veces más la probabilidad de presentar violencia de género, con un resultado estadísticamente significativo con un chi cuadrado de $p=, 000$. Aceptando la hipótesis alterna, demostrado en la campana de Gauss. Este hallazgo coincide con los resultados de OMS-OPS, UNICEF sobre el informe mundial sobre la violencia y la salud. Igualmente MINSA-Perú (Palomino Nancy, 2005; Barbera Herrera6 ${ }^{6}$ 2013) y (Rengifo Venegas Sofía)7 en 
donde se sostiene que la violencia de género tienen su origen en un orden social, donde prima la desigualdad de género sobre todo en los niveles socioeconómicos bajos donde es preponderante.

El 20,11 \% de la población inicio su relación sexual, con $11,11 \%$ mujeres y el $9 \%$ varones de 11 a 12 años de edad, a partir del $3^{\circ}$ a $5^{\circ}$ año de secundaria sobresalieron los varones. Estos resultados coinciden con los hallazgos internacionales de Rengifo Venegas ${ }^{7}$, Alvarado Thimeos ${ }^{8}$, Gonzales Electra ${ }^{9}$, Holguin ${ }^{10}$, Rincón Alexander ${ }^{11}$. En relación al concepto de liberación de las adolescentes mujeres, difiriendo con aquellos obtenidos por estudios de Rivera-Rivera $L^{12}$, Barbera Heredia ${ }^{13}$, Salazar $A^{14}$, donde el inicio de las relaciones sexuales fue mayor en los varones estando relacionado con el contexto sociocultural y el rol de género masculino predominante en nuestro país, parece explicar esta diferencia referente a la sexualidad y al concepto de masculinidad.

La OMS en el 2002 lanzo el informe mundial sobre la violencia y la salud, el sufrimiento "invisible" de las personas, la presente investigación halló 28 adolescentes, el 14,1 \% de estos presento violencia psicológica de los cuales el 6,7\% iniciaron sus relaciones sexuales, con un riesgo de 2.41 veces la probabilidad de presentar violencia psicológica; con una significación estadística de 0,032 presentando asociación entre las dos variables. Nuestro estudio comparte algunas similitudes con los trabajos de (Gonzales E9, 2012, Rangel Hernández M. 2012 ${ }^{15}$. Vega $\mathrm{Gea}^{16}$ 2013).

Con respecto a los que iniciaron su relación sexual tienen 1,87 veces más la probabilidad de riesgo de presentar violencia física, no existe asociación entre las dos variables al presentar $\mathrm{p}=0,197$ por lo tanto no existe significancia estadística, se acepta la hipótesis nula y se rechaza la hipótesis alterna, este resultado se debe a que la muestra es finita Se concluyó que de $19(9,6 \%)$ estudiantes presento violencia física, de los cuales el $4.04 \%^{8}$ inicio su relación sexual vs un $5.56 \%{ }^{11}$ que no inicio su relación sexual. Este hallazgos coinciden con la investigación de Vega Gea Elizabeth ${ }^{16}$ en España donde $63 \%$ de los estudiantes son victimizados físicamente por el padre, seguido del maltrato de la madre, encontrándose un porcentaje con inicio de su relación sexual.

Como ya se detalló en la revisión de la literatura, los resultados sobre los índices de prevalencia del fenómeno de la violencia sexual son diversos y dispares. El presente estudio halló que el inicio de la relación sexual fue forzado en el $24,7 \%$ para ambos sexos, con predominio (15,1\%) del sexo femenino y con una media de 0,27 vs el (9,6\%) para los varones con una media de 0,34. Este hallazgo nos demuestra que los estudiantes que iniciaron su relación sexual tienen 3,73 , veces más la probabilidad de riesgo de presentar violencia sexual (tabla $\mathrm{N}^{\circ}$ 4) con una asociación estadísticamente significativa en donde con un chi cuadrado con $p=0,002$. Además se demuestra que el $12.6 \%$ que presentaron violencia sexual, el 7.07 $\%$ iniciaron su vida sexual asociados a la disfunción familiar, viven solo con la madre $(4,3 \%)$ o padre con carencia del control parental y comunicación, resultados corroborados por las investigaciones de Rengifo V. Sofía ${ }^{7}$ (Perú), Alvarado Thimeos ${ }^{8}$ (Chile), Holguin M. Yuri ${ }^{10}$ (Colombia), el inicio de la RS tiene uno de los factores (no convivencia con los padres), según referencia bibliográfica internacionales de OMS-OPS la asociación de la historia de abuso sexual por un familiar o conocido, en la adolescencia por ser esta más vulnerable, además de no ser denunciado legalmente por múltiples razones como vergüenza, estigmatización, temor a represalias del violentador, sentimientos de culpabilidad y complejidad para denunciar, Estos investigadores señalaron porcentajes que varía de $5 \%$ al $40 \%$ de violencia sexual, con ella coexisten otros factores como la violencia familiar, drogas, poco conocimiento sobre sexualidad etc. En la misma tabla se observa que el $87.4 \%$ (173) que no presento violencia sexual, un $22.22 \%$ (44) inicio su relación sexual voluntariamente. La investigación de Rincón Alexander y Silva $\operatorname{Edgar}^{11}$ (Colombia) los adolescentes iniciaron su actividad sexual con personas mayores en 17 años para los varones vs 5 años mayor las parejas de las mujeres, además en ellos influyo la construcción tradicional de género sobre sus motivaciones demostrarse a sí mismo y confirmar su masculinidad y heterosexualidad.

El último objetivo de la investigación está dirigido al análisis violencia familiar, los estudiantes que iniciaron su relación sexual tuvieron 5,48 veces más la probabilidad de presentar el riesgo de violencia familiar, además tienen un resultado estadísticamente significativo con un valor de $\mathrm{p}=0.000$ es decir fuerte asociación entre las variables, así el $6.57 \%^{13}$ son mujeres el $10.1 \%{ }^{20}$ que presento violencia familiar. Este resultado coincide con los estudios internacionales de Rivera Rivera Leonor y colb ${ }^{12}$. (México), Barbera Heredia Esther ${ }^{13}$ (España), Parra Villaroel Jaime (Chile $\left.{ }^{17}\right)$, demostraron que el inicio de la actividad sexual en mayor cuando existe violencia intrafamiliar conjuntamente a la agresión psicológica $(22,5 \%)$, vivir solo con su madre, mala comunicación 
familiar, mala relación padre - hijo, ausencia del padre, etc; demuestran la influencia de la violencia familiar en el inicio sexual, así mismo comprobadas en las investigaciones nacionales de (Rengifo Venegas Sofía ${ }^{7}$, Mayorga H, Elizabeth ${ }^{18}$, Salazar. A y colb ${ }^{19}$. Gamarra Tenorio Patricia $\left.{ }^{20}\right)$. está asociado con la concepción patriarcal de control y pertenencia sobre los hijos y la falta o mala comunicación entre los padres-hijos en las relaciones de poder de los padres sobre ellos, además de la aceptación social generalizada de las normas de corrección a través del castigo.

\section{CONCLUSIÓN}

En base a los resultados obtenidos concluimos que los adolescentes que iniciaron su relación sexual tienen alta probabilidad de riesgo de violencia y asociación altamente significativa $(p ; 0,00)$ con la violencia familiar; seguido de la violencia sexual, La violencia psicológica ocupa el tercer lugar y en último lugar violencia física, ambas son factores de riesgo pero no tienen asociación significativa .

Las variables con asociación significativa con la violencia de género son: inicio de las RS, año que cursa, violencia psicológica, sexual y familiar
Mientras que las variables sexo, edad, lugar donde viven, con quién viven y violencia física no están asociadas a la violencia de género. Las variables con OR: >1: Inicio RS, edad, con quien viven, los 4 tipos de violencia tienen veces más la probabilidad de presentar el riesgo de violencia de género.

No son factor de riesgo por tener OD $<1$ las variables sexo, lugar donde viven y año que cursan.

Agradecimiento : Queremos agradecer al Dr. Jhony De La Cruz Vargas de manera especial por su paciencia, tiempo y apoyo para la realización de este artículo de investigación.

Financiamiento: Autofinanciado.

Conflicto de interés: Los autores declaran no tener conflictos de interés en la publicación de este artículo.

Recibido: 05 de diciembre del 2016

Aprobado: 30 de diciembre del 2016

\section{REFERENCIAS BIBLIOGRÁFICAS}

1. Organización Mundial de la Salud. Centro de prensa $N^{\circ} 239$. Enero 2016. INTERNET. [citado: $1^{\circ}$ Marzo 2016] Disponible en: http://www.who. int/mediacentre/factsheets/fs239/es/.

2. Organización Mundial de la Salud. Serie Género y Salud Pública Modelo Integral para Abordar la VIF. Programa Mujer, Salud y Desarrollo PAHO/WHO. [citado: 3 Marzo 2016]. Disponible en: https://es.search. yahoo.com/search? $p=$ Organizaci\%C3\%B3n++Mundial+de+la+Salud.+Serie+G\%C3\%A9nero+y+Salud+P\%C3\%BAblica+Modelo+Integral+para+Abordar+la+VIF.+Programa+Mujer\%2C+Salud+y+DesarroIlo+\%E2\%80\%93++PAHO\%2FWHO\&ei=UTF-8\&fr $=$ moz35

3. Center for health and gender Equity. Para acabar la violencia contra la mujer. Population Report Serie 1, N 11, Vol XXVII, N 4. Diciembre 4. 1999. (página en internet) [citado:

4. Marzo 2016] Disponible en: http://pdf.usaid.gov/pdf_docs/Pnack420.pdf

5. Encuesta demográfica y de salud familiar ENDES -INEI. Lima. 2014. [citado 10 marzo 2016].Disponible en: https://www.google.com. pe/?gws_rd=ssl\#q=Encuesta+Demogr\%C3\%A1fica+y+de+Salud+Familiar+2014

6. Barberá Heredia Esther, Ferrer Cascales Rosario, Navarro-Pertusa Esperanza, Grupo de iguales e iniciación sexual adolescente diferencias de género 2010. Andalucía. España. International journal of clinical and health psychology, ISSN 1697-2600, Vol. 6, №. 1, 2010, págs. 79-96. [Citado: 19 Abril 2016]. Disponible en. https://dialnet.unirioja.es/servlet/ articulo?codigo $=1987281$
7. Rengifo Venegas, Sofía. Revista Médica Panacea. Vol. 4, Núm. 1 (2014). Internet. [Citado: 19 Abril 2016]. Disponible: www.revpanacea.pe/index. php/RMP/article/view/82

8. Alvarado Thimeos Julia 2013. Educación sexual preventiva en adolescentes de Santiago de Chile. Educación sexual preventiva en adolescentes - Contextos, № 29, 2013, 25-42. [citado: 14 Abril 2016] Disponible en: http://e-spacio.uned.es/fez/eserv/tesisuned:Educacion- Oealvarado/ ALVARADO_THIMEOS_Julia_Eliana_Tesis.pdf

9. González E, Molina T, Montero A, Martínez V. Factores asociados al inicio sexual en adolescentes de ambos sexos de nivel socioeconómico medio-bajo de la Región Metropolitana. Rev. chil. obstet. ginecol. [Revista en la Internet]. 2013.78 (1):4-13. .[Citado 13 Agosto 2016]. [Citado 14 Abril 2016] Disponible en: http://www.revistasochog.cl/articulos/ver/598

10. Holguín M Yuri. Mendoza T. Luis Alfonso, Esquivel T Claudia Marcela, Sánchez M, Ricardo. Daraviña B. Andrés Felipe. Acuña P, María.

Factores asociados al inicio de la actividad sexual en adolescentes de Tuluá, Colombia. 2012 Rev. chil. Obstet. Ginecol. Vol 78 N³.Santiago de Chile. 2013. [Citado: 17 Abril 2016] . Disponible en: http://www.scielo. cl/scielo.php?script=sci_arttext\&pid=S0717-75262013000300007 http:// dx.doi.org/10.4067/S0717-75262013000300007.

11. Rincón Alexander, Silva Edgar. Primera relación sexual en adolescentes escolares de Montelíbano (Córdoba, Colombia): Un análisis desde sus relatos. Revista Colombiana de Enfermería. Vol 8, Año 8 (2013) p. 61-73. Recibido: 2013-06-6; aprobado: 2013-07-1. Internet. [Citado: 18 Abril 2016]. Disponible: http://revistas.unbosque.edu.co/index.php/RCE/article/view/547 
12. Rivera Rivera Leonor, Margarita de los Santos Lilia, Leyva-López Ahidée, et al. 2012. Inicio de relaciones sexuales con penetración (IRSP) y factores asociados en chicos y chicas de México de 14-19 años de edad con escolarización en centros públicos. Gaceta Sanitaria, Volume 30, Issue 1, January-February 2016, Pages 24-30. [citado: 16 Abril 2016]. Disponible en: http://www.sciencedirect.com/science/article/pii/ S0213911115001727

13. Barberá Heredia Esther, Ferrer Cascales Rosario, Navarro-Pertusa Esperanza, Grupo de iguales e iniciación sexual adolescente diferencias de género 2010. Andalucía. España. International journal of clinical and health psychology, ISSN 1697-2600, Vol. 6, №. 1, 2010, págs. 79-96. [Citado: 19 Abril 2016]. Disponible en. https://dialnet.unirioja.es/servlet/ articulo?codigo $=1987281$

14. Salazar A, Santa María A, Solano I , Lázaro K, Arrollo S, Araujo V, Luna D, Echazu C. Conocimientos de sexualidad, inicio de relaciones sexuales y comunicación familiar en adolescentes de instituciones educativas nacionales del distrito de el Agustino, Lima-Perú. 2010. [revista en internet].201017 (2).[citado 20 Abril 2016]. Disponible en: http://www.medicina.usmp.edu.pe/horizonte/2007_II/Art3_Vol7_N2.pdf

15. Rangel Hernández Monserrat, Martínez Ramón Yanelly. Perea Ortiz María Guadalupe. Gallegos Torres Ruth, M y colb. Características emocionales de los adolescentes que han iniciado una vida sexual activa. Querétaro. México, 2012. Lux Médica. Año 10 №30 Julio- Agosto 2012. PP 23 - 30 [Internet] . [Citado: 18 Abril 2016]. Disponible en: https://issuu.com/ editorialuaa/docs/luxmedica30

16. Vega Gea Esther María. La violencia sexual en adolescia: Naturaleza del fenómeno y factores asociados. Universidada de Cordova. España. 2013. [Citado: 15 Abril 2016] Disponible en: http://hdl.handle. net/10396/11954. http://helvia.uco.es/xmlui/handle/10396/11954.

17. Parra Villarroel Jaime 2010. Comportamiento sexual en adolescentes y su relación con variables biosociales realizado en la Institución de Salud
"Concepción" en el Departamento de Obstetricia en Chile. Perinatol Reprod Hum.2010; 24(1):7-19. [citado: 18 Abril 2016]. Disponible en: www. medigraphic.com/pdfs/inper/ip-2010/ip101b.pd

18. Mayorga H, Elizabeth, Ñique N, Máximo, Franco Roxana, Servan N Cynthia, Rojas Eddy, Arango Karol, Pérez Del Águila José. " Comportamiento Sexual de riesgo en escolares de secundaria de Lima Sur.2014. Rev. de psicología. Facultad de Psicología UNFV.[Revista en Internet] Disponible en: http://www.rppsicometria.com.pe/catedra/index.php/RCVFAPS/article/view/107/73 [.citado 19 Abril 2016. ]

19. Salazar A, Santa María A, Solano I , Lázaro K, Arrollo S, Araujo V, Luna $D$, Echazu C. Conocimientos de sexualidad, inicio de relaciones sexuales y comunicación familiar en adolescentes de instituciones educativas nacionales del distrito de el Agustino, Lima-Perú. 2010. [revista en internet].201017 (2).[citado 20 Abril 2016]. Disponible en: http://www.medicina.usmp.edu.pe/horizonte/2007_II/Art3_Vol7_N2.pdf

20. Gamarra Tenorio Patricia, lannacone José. Factores asociados que influyen en el inicio de actividad sexual en adolescentes escolares de villa San Francisco-Santa Anita, Lima-Perú, 2010. The Biologist (Lima). Vol. 8, №1, enero-junio 2010. The Biologist, ISSN-e 1816-0719, Vol. 8, №. 1, 2010, págs. 54-72. [Citado: 20 Abril 2016]. Disponible en: https://dialnet.unirioja.es/servlet/articulo?codigo $=3990005$

21. Violencia sexual en América y el Caribe: análisis de los datos secundarios, Versión Marzo 2010.p 38-39. [Citado 27 Mayo 2016] Disponible en: http://www.oas.org/dsp/documentos/Observatorio/violencia_sexual_ la_y_caribe_2.pdf

22. Sustainable Development Goals. New York: United Nations; 2015. Available. [Citado: mayo 2016 Disponible en: http://www.un.org/sustainabledevelopment/sustainable-development-goals/].

Consulte la Versión Electrónica de la Revista:

Facultad de Medicina Humana Universidad Ricardo Palma

http://revistas.urp.edu.pe/ojs/index.php/RFMH<smiles>C1CCCC1</smiles>

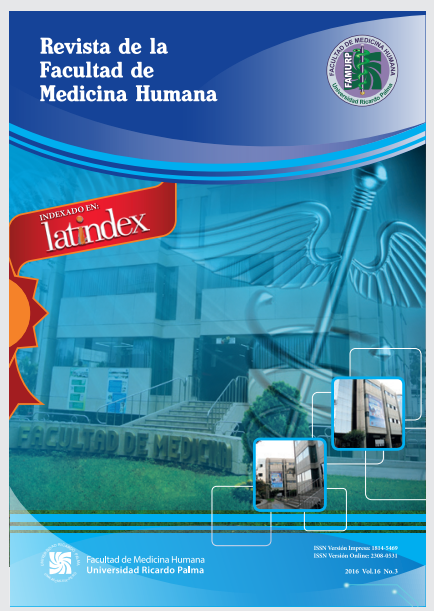

